\title{
Breastfeeding support practices in designated workplaces in the Breede Valley sub-district, Western Cape, South Africa
}

\author{
L Daniels, PhD (Nutr Sc); L M Du Plessis, PhD (Nutr Sc); X Mbhenyane, PhD (Nutr) \\ Division of Human Nutrition, Department of Global Health, Faculty of Medicine and Health Sciences, Stellenbosch University, Cape Town, \\ South Africa
}

Corresponding author: L Daniels (ldaniels@sun.ac.za)

\begin{abstract}
Background. Globally, mothers have identified employment as one of the leading barriers to exclusive and continued breastfeeding. The workplace and employment setting has been highlighted as one component of an enabling environment for breastfeeding.

Objectives. To assess breastfeeding support practices in designated workplaces in the Breede Valley sub-district, Western Cape, South Africa.

Methods. A quantitative, cross-sectional design study was used. An online survey was conducted amongst human resource managers and/or company managers to determine breastfeeding support practices in designated workplaces.

Results. Fourteen participants completed the online survey. Breastfeeding support practices in designated workplaces were limited and inadequate, with few supportive breastfeeding practices. Onsite or nearby crèche facilities $(n=2 ; 14.3 \%)$, breastfeeding counsellors $(n=1 ; 7.1 \%)$, promotion of the benefits of breastfeeding to employees $(n=2 ; 14.3 \%)$ and provision of private space for expressing $(n=2 ; 14.3 \%)$ were not common practices. Workplace breastfeeding policies $(n=4 ; 28.6 \%)$ were not common practice, and were mostly found in the public sector. Forty-three percent of workplaces $(n=6 ; 42.9 \%)$ did not provide time for expressing at work.

Conclusion. There is an urgent need to create advocacy regarding the benefits of breastfeeding support in the workplace for employers, employees and society as well as the breastfeeding rights of women in the workplace. The legislated breastfeeding break times need to be monitored to ensure better compliance in workplaces.
\end{abstract}

S Afr J Child Health 2020;14(2):94-98. https://doi.org/10.7196/SAJCH.2020.v14i2.1679

The workplace and employment setting were highlighted in the 2016 Lancet series as one component of an enabling environment for breastfeeding. ${ }^{[1]}$ Globally, mothers have identified employment as one of the leading barriers to exclusive and continued breastfeeding. ${ }^{[2]}$ Working mothers often find it challenging to combine and continue breastfeeding with full-time employment, especially if workplace support is absent. ${ }^{[3]}$ If women are not supported, they often turn to formula feeding when returning to work. Globally, only $37 \%$ of infants under 6 months are exclusively breastfed. ${ }^{[2]}$ The World Health Assembly (WHA) has set a global target to increase the rate of exclusive breastfeeding (EBF) in the first 6 months of life to at least $50 \%$ by $2025 .{ }^{[4]}$ For a mother to reach 6 months of EBF, government direction is required as well as support from families, communities, the health system and workplaces. ${ }^{[5]}$

In South Africa (SA), EBF rates have increased over the past decade, but remain low at $32 \%$, below the WHA target. ${ }^{[6]}$ With more women entering the workforce, there is a need for extended support to mothers in the workplace to enable them to sustain their breastfeeding practices and improve EBF and breastfeeding rates in the country. Workplaces are excellent settings for the implementation of interventions that assist the initiation and the continuation of $\mathrm{EBF}^{[7]}$ Breastfeeding supportive practices in the workplace can include: developing policies to support breastfeeding mothers in the workplace; providing a private area (other than a bathroom) for women to breastfeed or express milk; allowing time and flexibility to express breastmilk at work; providing working mothers with options when returning to work, such as working parttime, job sharing or extending maternity leave; providing onsite or nearby childcare facilities at work; and offering supportive lactation management services. ${ }^{[8]}$ Several publications have shown that the presence of workplace lactation support enhances working mothers' ability to continue breastfeeding along with employment. ${ }^{[3,7,9,10]}$

Little evidence is currently available in SA regarding breastfeeding supportive practices within the workplaces as well as the implementation and practice of legislated breastfeeding break times that mothers are entitled to. Rollins et al. ${ }^{[1]}$ inspired the research reported here, which aimed to explore and understand the workplace environment with regard to support for breastfeeding. Owing to limited evidence in the SA context, the present research set out to assess current breastfeeding support practices in designated workplaces in the town of Worcester, situated in the Breede Valley sub-district, Western Cape (WC) Province. Support from employers and workplaces can significantly influence breastfeeding duration and exclusivity rates among employed mothers. ${ }^{[9]}$ Awareness of current breastfeeding support practices in designated workplaces can inspire focused strategies to improve such practices.

\section{Methods}

A quantitative, descriptive, cross-sectional study was conducted. The Breede Valley sub-district of WC, SA, was used as the setting as it represents workplace linkages to local, regional, provincial (e.g. government departments) as well as national level entities (retail stores and large commercial food companies). 
The study population included private and public designated workplaces. Designated workplaces are businesses with more than 50 employees. ${ }^{[1]}$ Workplaces were selected from 3 categories: (i) the private designated business sector including producers (farmers), manufacturers and distributors; (ii) the private designated business sector including traders (retail shops); and (iii) the public sector.

A list was compiled of all designated workplaces in the sub-district. Various sources were used to compile the list, e.g. business forum administrative officer, government websites and human resource officers. All workplaces were contacted telephonically to verify that they were designated workplaces and to obtain contact details of human resource managers (HRMs). A total of 38 designated workplaces were included in the sample.

A baseline online survey was conducted during September and November 2017 with HRMs and/or company managers (director, chief executive officer) of the various designated workplaces. HRMs and/or company managers were selected as they were in a position to provide information of company policies as well as report any barriers experienced or foreseen or any enablers within their setting. They are also the individuals who can potentially influence workplace environment and policies.

The first author (LD) developed the questionnaire for the online survey. Baseline data collected included aspects of breastfeeding support interventions, e.g. maternity leave benefits, paternity leave benefits, workplace policies, wellness, occupational programmes, nearby or onsite crèche facility, provision of private space and breastfeeding time. Content validity was tested by circulating the survey questionnaire to three experts in the field of infant and young child nutrition and human resources management prior to the pilot study. The face validity of the survey questionnaire was tested during the pilot study.

The SurveyMonkey (SVMK; USA) software programme was used to conduct the online survey. The online questionnaire was available in English as it was expected that most managers would be able to read and understand English. The survey link was sent via email to all HRMs or company managers and included a motivation on why the survey was of importance and the anticipated value of participants' input. Participants had access to the survey for six weeks. Reminder emails were sent every two weeks to promote participation. Owing to the initial low response rate, access to the survey was extended by another seven weeks. In addition, the researcher contacted workplaces telephonically and visited sites to further encourage participation. An option to complete the survey in 'hard copy' was also provided to workplaces; these were manually entered in the SurveyMonkey programme by the researcher.

Data were exported from SurveyMonkey into Excel (Microsoft; USA) and a statistician assisted with data analysis. Summary statistics were used to describe the variables. Distributions of variables were presented with histograms and/or frequency tables. Medians or means were used as measures of central location for ordinal and continuous responses and standard deviations and quartiles as indicators of spread.

Ethics approval (ref. no. S17/04/089) was granted by the Health Research Ethics Committee, Stellenbosch University. Permission to conduct the online survey with HRMs from public-sector workplaces was obtained from the relevant departmental ethics committees. Participation in the online survey was voluntary. Informed consent was acquired at the start of the survey, when participants were asked to accept or decline participation in the survey. Once participants accepted, they were able to proceed with the survey. A link was also provided where a copy of the participant information leaflet and consent form could be obtained. To ensure anonymity, participants were assigned a code; no company or participant names were collected.

\section{Results}

\section{Characteristics of participants}

A response rate of $37.0 \%(n=14 / 38)$ was obtained. The results presented here focused on baseline breastfeeding supportive practices at workplaces. The characteristics of participants are reflected in Table 1 . Participants were more or less evenly distributed between the public $(n=4)$, retail $(n=3)$ and manufacturing $(n=3)$ sectors.

The provision of workplace structural support practices for breastfeeding (e.g. space, crèche facility, policies) are outlined in Table 2. Onsite or nearby crèche facilities, breastfeeding counsellor, promoting the benefits of breastfeeding to employees and private space provided for breastfeeding employees were not common practices at workplaces. Open-ended comments on the main reasons for not providing space included lack of space and infrastructure $(n=8)$. Similarly, workplace breastfeeding policies were not common

\section{Table 1. Characteristics of participants}

\begin{tabular}{ll}
\hline Variable $(N=14)$ & $n(\%)^{\star}$ \\
\hline Gender & $3(21.4)$ \\
Male & $11(78.6)$ \\
Female & $44.7(10.1)$ \\
Age, mean (SD) & \\
Position & $11(78.6)$ \\
Human resource manager & $1(7.1)$ \\
Chief executive officer & $1(7.1)$ \\
General manager & $1(7.1)$ \\
Human resource and facility manager & $7.5(7.0)$ \\
Years employed, mean (SD) & \\
Relationship status & $12(85.7)$ \\
Married & $1(7.1)$ \\
Living together & $1(7.1)$ \\
Divorced/separated & $12(85.7)$ \\
Own children & $1(7.1)$ \\
Grandchildren & \\
Employer type & $3(21.4)$ \\
Retail & $4(28.6)$ \\
Public/government & $3(21.4)$ \\
Manufacturer & $1(7.1)$ \\
Private healthcare & $3(21.4)$ \\
Other: welfare, security, non-profit company & \\
$*$ Unless otherwise specified. &
\end{tabular}

Table 2. Workplace structural support provided for breastfeeding $(N=14)$

\begin{tabular}{ll}
\hline Structural support for breastfeeding & $n(\%)$ \\
\hline Occupational health programme & $8(57.1)$ \\
Onsite/nearby crèche & $2(14.3)$ \\
Private space and room & $2(14.3)$ \\
Written breastfeeding policy & $4(28.6)$ \\
Refrigerator for breastmilk storage & $5(35.7)$ \\
Breastfeeding counselling for staff & $1(7.1)$ \\
Educational material for pregnant and new mothers & $5(35.7)$ \\
Educational material for expecting fathers & $5(35.7)$ \\
Routinely promote benefits of breastfeeding to employees & $2(14.3)$
\end{tabular}


practice, and were found more in the public sector.

A structural breastfeeding support score out of nine was determined and the mean structural support scores for breastfeeding were compared between the public and private sectors. Fig. 1 reflects the mean structural support score of five for the public sector workplaces and one for the private sector workplaces, indicating that the public sector was more likely to provide structural support for breastfeeding. Owing to the small sample size, no further statistical tests could be performed.

Fig. 2 reflects workplace time support practices for breastfeeding. Open-ended responses for the reasons for not providing time included no formal request received from employees $(n=2)$, time pressure $(n=1)$, unaware that it is mandatory to provide time $(n=1)$ and not prioritised $(n=1)$ within the workplace.
A time support score out of seven elements was determined for the workplaces. Fig. 3 indicates a higher mean time support score of four for the public sector and three for the private sector

The majority of participants $(93 \% ; n=13)$ indicated having knowledge of the Code of Good Practice (CGP) on the Protection of Employees during pregnancy and after the birth of a child (part of the Basic Conditions of Employment Act (BCEA)). The need for more information on how to support breastfeeding women at work was voiced by more than half $(57 \% ; n=8)$ of the participants. Open-ended comments on the needs identified by participants to accommodate breastfeeding at work included information, education, training on the benefits of breastfeeding support $(n=5)$, a breastfeeding policy and communication $(n=1)$ and a space regulatory framework or standard $(n=1)$

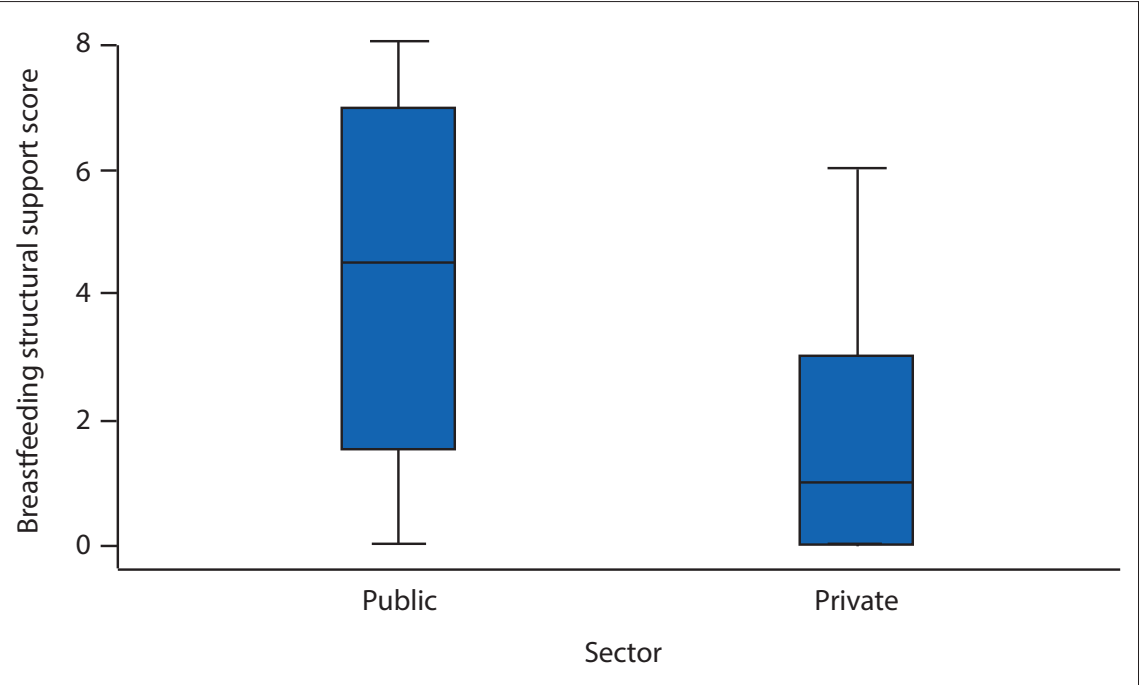

Fig. 1. Structural breastfeeding support score between the public and private sectors.

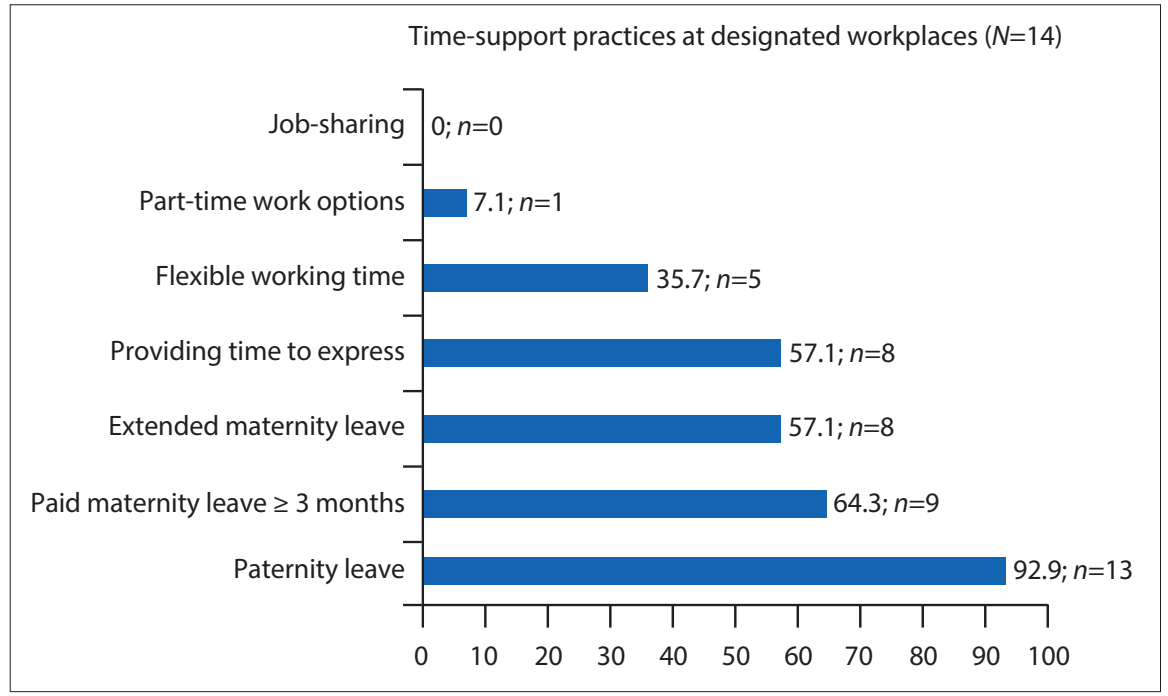

Fig. 2. Workplace time-support practices.

\section{Discussion}

The aim of the study was to assess breastfeeding support practices in designated workplaces. The findings of the baseline survey indicate that structural and time support practices for breastfeeding in designated workplaces are limited and inadequate.

The presence of a written breastfeeding policy was not common practice and was mainly present in public-sector workplaces. In Colorado, Dunn et al., ${ }^{[12]}$ and more recently Hojnacki et al. ${ }^{[13]}$ in Michigan, similarly found that few workplaces had written breastfeeding policies ( $4 \%$ and $3 \%$, respectively). A survey conducted among diverse organisations in South Africa found that only $16 \%(n=5)$ of them had a written breastfeeding policy in place. Reasons provided for the lack of written breastfeeding policies by the survey respondents included lack of demand, possible ignorance on the part of employee representatives, and unsuitable buildings that would make policy implementation impossible. ${ }^{[14]}$ There are no explicit guidelines for employers relating to breastfeeding policies in the workplace in SA.

Formal policies have the benefits of providing and establishing standard guidelines and expectations for workplace breastfeeding support. Policies at the level of the employer and government are essential for creating a supportive environment for breastfeeding. Also, when formal policies are in place, it does not guarantee that support is provided and does not indicate how the support is experienced. Therefore, interpersonal communication is important to consider together with workplace support for breastfeeding, as echoed by Anderson et al. ${ }^{[15]} \mathrm{A}$ recent review of workplace lactation accommodation found that having workplace lactation policies was not consistently associated with breastfeeding duration. ${ }^{[16]}$ The results were mixed, with Dabritz et al. ${ }^{[17]}$ indicating a significant association with any breastfeeding at 6 months, and Bai and Wunderlich ${ }^{[18]}$ who found no significant association between workplace policies and EBF at 6 months. Policies alone will also not change discouraging organisational attitudes towards breastfeeding mothers. Therefore, the need for education to steer the attitude to more favourable actions must be considered.

The present study revealed that provision of a private space was not a common practice. Dodgson et al. ${ }^{[19]}$ mailed surveys to 19 maternity hospitals in Hong Kong. Similarly to our findings, only a low percentage $(26 \% ; n=5)$ of the hospitals 


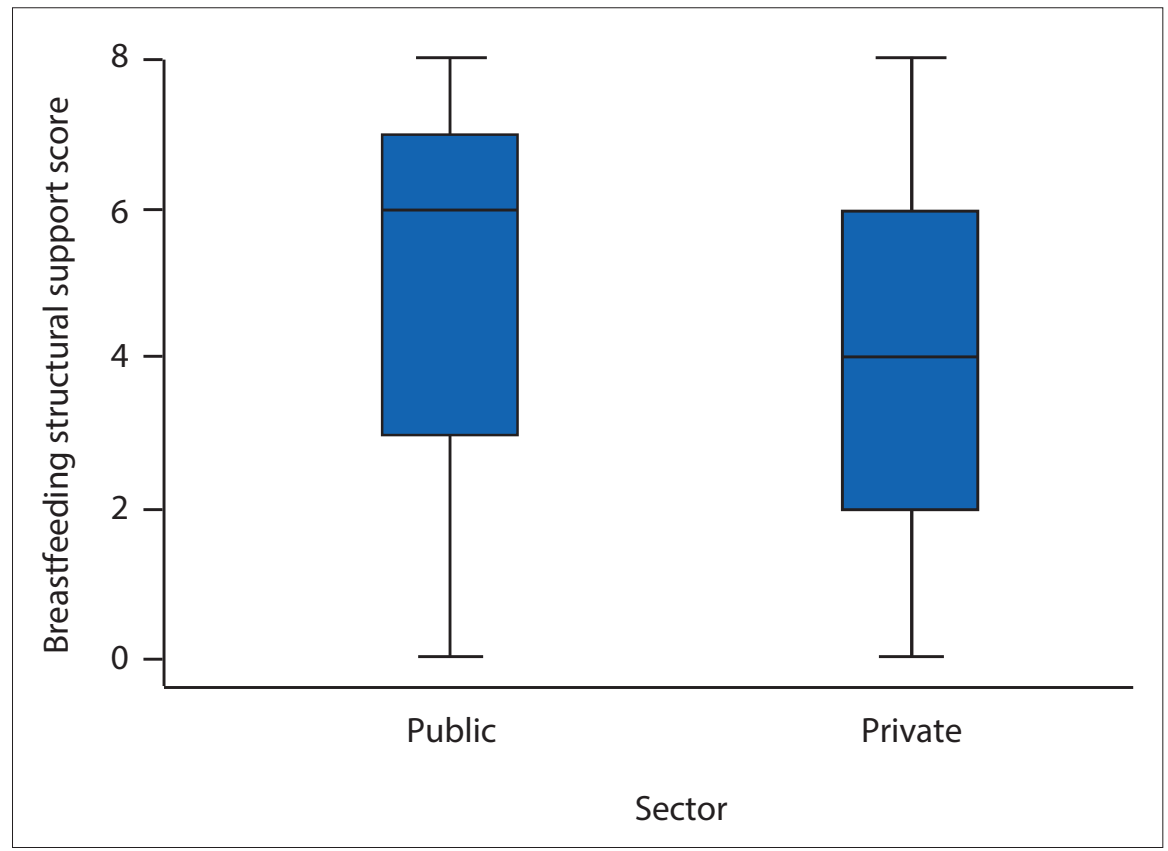

Fig. 3. Breastfeeding time support score between the public and private sectors.

had a private room with a lockable door available for breastfeeding mothers. Hojnacki et al., ${ }^{[14]}$ however, indicated a higher percentage $(78 \%)$ of companies having non-restroom space available for expressing in Michigan. This figure is higher than those of other reported studies by Dunn et al. ${ }^{[12]}$ in Colorado and Soomro et al. ${ }^{[21]}$ in Pakistan, in which only $34 \%$ and $6 \%$ respectively possessed this amenity. Important to note is that Hojnacki et al..$^{[13]}$ found that when looking at a designated space solely for breastfeeding or expressing, only $32 \%$ of respondents had such a facility available. Weber et al. ${ }^{[21]}$ in Sydney, Australia, found that $19 \%$ of health service workplaces had designated space available, solely for breastfeeding.

The main reason stated for not providing a dedicated space for breastfeeding or expressing was the lack of space and infrastructure, which is a real-time situation in many workplaces. The Business Case for Breastfeeding, a US Department of Health and Human Services Office of the Women's Health Project, provides useful resources, guidance and tips for workplaces that need to overcome space challenges. ${ }^{[22]}$ It is important that workplaces receive support from Department of Health officials, who can assist them in creating a breastfeeding private space (designated or flexible space) and refer them to resources which can provide guidance on how to overcome the challenge of lack of space.

Other reasons for lack of providing a designated private space might be linked to misconceptions about the cost implications of setting up a private space, or lack of knowledge about what breastfeeding support entails, and what health and economic benefits follow from higher breastfeeding rates. The benefits of providing a working environment conducive to breastfeeding outweigh the costs. If breastfeeding is supported in the workplace, women are more likely to return to work, which contributes to women maintaining their job skills, as well as reducing staff turnover. ${ }^{[7]}$ Workplaces may, however, view breastfeeding as a non-workplace issue, and misconceptions about providing support in terms of space might lead to decreased productivity among employees. A study by WittersGreen $^{[23]}$ showed that employers believed that breastfeeding was not a workplace issue and that mothers would miss more work, by choosing to stay home with their infant. The provision of breastfeeding space and time is in fact a low-cost intervention that has been shown to reduce absenteeism and improve work performance, commitment and retention among staff. ${ }^{[2]}$

Fifty-seven percent $(n=8)$ of workplaces reported providing time for expressing at work, with the remainder $(43 \% ; n=6)$ not providing time for expressing. Studies from Hong Kong ${ }^{[19]}$ and Pakistan ${ }^{[20]}$ assessed the prevalence of workplace breastfeeding facilities and found that a much lower percentage of workplaces offered breastfeeding breaks to working mothers (11\% and $15 \%$, respectively). In contrast, a study in Michigan found that a high percentage $(73 \%)$ of companies provided breastfeeding time. ${ }^{[13]}$
The high percentage of workplaces in SA not providing time for expressing is concerning. The CGP on Protection of Employees during pregnancy and after the birth of a child (part of the BCEA 1997, amended in 2014), stipulates that arrangements should be made for employees who are breastfeeding to have two 30-minute breaks per day for breastfeeding or expressing for the first 6 months of a child's life. ${ }^{[2]}$ Most workplaces in the present survey, however, indicated that they were aware of the CGP, which again brings attention to either a lack of practice and implementation ${ }^{[14]}$ or a lack of awareness of the legislation. Alternatively, the respondents might have indicated the desirable answer during the survey. Furthermore, the percentage of workplaces that indicated providing time for expressing at work might have been overreported, owing to respondents that may have confused providing time for breastfeeding, with routine breaks. If breastfeeding legislation is monitored in SA, workplaces will most likely focus more on the implementation and practice thereof. A survey conducted in SA found that $13 \%$ of workplaces provided no breastfeeding breaks, ${ }^{[14]}$ which is much lower than the findings in the present survey. It is evident in SA that there are low levels of compliance with breastfeeding support laws. ${ }^{[14]}$ The SA survey by Martin-Wiesner ${ }^{[14]}$ and the Bangladeshi mothers-at-work case study, similarly identified barriers relating to low enforcement of breastfeeding laws and policies. ${ }^{[5]}$

Almost $60 \%$ of workplaces indicated a need to receive more information on how to support breastfeeding women at work, which may indicate lack of knowledge of how to support breastfeeding at the workplace, but also a lack of willingness to do so. There is an urgent need for advocacy among these categories of workplaces pertaining to the benefits of breastfeeding support for employees, workplaces and greater society as well as women's breastfeeding rights in the workplace. It is also considered important that not only employers, but also employees, be educated about their breastfeeding rights, so that they can request them and take up these rights within the workplace. SA has a high and growing mobile phone penetration rate, with 15 million users of social media platforms and 13 million users doing so purely from mobile phones. ${ }^{[25]}$ More media campaigns and social media movements must be utilised to create increased awareness of women's breastfeeding rights within communities and society. A survey of 715 working mothers employed in a manufacturing plant in Taiwan found that 
a breastfeeding room with a dedicated space (odds ratio (OR) 2.38) and the use of breastfeeding breaks (OR 61.6) were significant predictors of continued breastfeeding for more than 6 months after returning to work. ${ }^{[26]}$ Therefore, with SA's low EBF rate of $32 \%$, it is important that more focus be placed on workplace support for breastfeeding in terms of providing private space, time and support.

\section{Conclusion}

The workplace has been identified as a leading barrier to exclusive and continued breastfeeding. The present study found that breastfeeding support practices in designated workplaces are limited and inadequate, with few supportive breastfeeding practices. There is an urgent need to create advocacy among workplaces regarding the benefits of breastfeeding support in the workplace for the employer, employees and society, and the breastfeeding rights of women in the workplace. Employers need to be educated on their role and responsibilities in this regard. There is also a need for legislated breastfeeding break times to be monitored so that implementation and practice can improve. The present baseline survey results will be used to inform the development of a workplace model for supporting breastfeeding in the workplace. Further research can be conducted in other research settings and can include a more diverse group of participants to gain their perspectives of workplace breastfeeding support practices. The small sample size achieved in the online survey limits the generalisability of the findings to other settings.

\section{Declaration. None.}

Acknowledgements. The researchers would like to acknowledge and thank the participants of the survey.

Author contributions. LD developed the idea and the protocol for this research study, planned the research, undertook data collection (with the assistance of trained research assistants), analysed the data with the assistance of a statistician, interpreted the data and drafted the article. XM and LdP provided input at all stages and revised the protocol and article.

Funding. Funding from the National Research Foundation was received for this research.

Conflicts of interest. None.

1. Rollins NC, Bhandari N, Hajeebhoy N, et al. Why invest, and what it will take to improve breastfeeding practices? Lancet 2016;387(10017):491-504. https:// doi.org/10.1016/s0140-6736(15)01044-2

2. Victora CG, Bahl R, Barros AJD, et al. Breastfeeding in the 21st century: Epidemiology, mechanisms and lifelong effect. Lancet 2016;387(10017):475-490. https://doi.org/10.1016/s0140-6736(15)01024-7

3. Yimyam S, Hanpa W. Developing a workplace breastfeeding support model for employed lactating mothers. Midwifery 2014;30(6):720-724. https://doi. org/10.1016/j.midw.2014.01.007

4. World Health Organization. Global targets 2025. To improve maternal, infant and young child nutrition. https://www.who.int/nutrition/topics/nutrition globaltargets2025/(accessed 5 February 2019).

5. United Nations Children's Fund. Let's make it work! Breastfeeding in the workplace - Using communication for development to make breastfeeding possible among working mothers. New York: UNICEF, April 2018. https:// www.healthynewbornnetwork.org/hnn-content/uploads/Mother_BabyFriendly WorkplaceInitiativeC4D web1_002 .pdf (accessed 5 February 2019).

6. South Africa Demographic and Health Survey 2016: Key Indicators Report. Pretoria: Statistics South Africa, 2016. https://www.statssa.gov.za/publications/ Report\%2003-00-09/Report\%2003-00-092016.pdf (accessed 5 February 2019).
7. Ortiz J, McGilligan K, Kelly P. Duration of breast milk expression among working mothers enrolled in an employer-sponsored lactation program. Paediatr Nurs 2004;30(2):111-119.

8. Stewart-Glenn J. Knowledge, perceptions, and attitudes of managers, coworkers, and employed breastfeeding mothers. AAOHN J 2008;56(10):423431. https://doi.org/10.3928/08910162-20081001-02

9. Balkam JA, Cadwell K, Fein SB. Effect of components of a workplace lactation program on breastfeeding duration among employees of a public-sector employer. Matern Child Health J 2011;15(5):677-683. https://doi.org/10.1007/ s10995-010-0620-9

10. Kozhimannil KB, Jou J, Gjerdingen DK, McGovern PM. Access to workplace accommodations to support breastfeeding after passage of Affordable Care Act. Womens Health Issues 2016;26(1):6-13. https://doi.org/10.1016/j. whi.2015.08.002

11. National Department of Labour, South Africa. Employment Equity Act, 1998 (Act No. 55 of 1998). Employment Equity Act. Government Gazette No. 19370:1323. http://www.labour.gov.za/DOL/DOL/downloads/legislation/acts/ employment-equity/eegazette2015.pdf (accessed 11 February 2019).

12. Dunn BF, Zavela KJ, Cline AD, Cost PA. Breastfeeding practices in Colorado businesses. J Hum Lact 2004;20(2):170-177. https://doi. org/10.1177\%2F0890334404263739

13. Hojnacki SE, Bolton T, Fulmer IS, Olson BH. Development and piloting of an instrument that measures company support for breastfeeding. J Hum Lact 2012;28(1):20-27. https://doi.org/10.1177/0890334411430666

14. Martin-Wiesner, P. 2018. A Policy-friendly environment for breastfeeding: A review of South Africa's progress in systematising its international and national responsibilities to protect, promote and support breastfeeding. Johannesburg: DST-NRF Centre of Excellence in Human Development, January 2018. https://www.wits.ac.za/media/wits-university/research/coehuman/documents/Breastfeeding\%20policy\%20review.pdf (accessed 5 February 2019).

15. Anderson J, Kuehl RA, Mehltretter Drury SA, et al. Policies aren't enough J Hum Lact 2015;31(2):260-266. https://doi.org/10.1177/0890334415570059

16. Hillard ED. A review of worksite lactation accommodations. Occupational health professionals can assure success. Workplace Health Saf 2017;65(1):3344. https://doi.org/10.1177/2165079916666547

17. Dabritz HA, Hinton BG, Babb J. Evaluation of lactation support in the workplace or school environment on 6-month breastfeeding outcomes in Yolo County, California. J Hum Lact 2009:25(2):182-193. https://doi. org/10.1177/0890334408328222

18. Bai Y, Wunderlich SM. Lactation accommodation in the workplace and duration of exclusive breastfeeding. J Midwifery Womens Health 2013;58(6):690-696. https://doi.org/10.1111/jmwh.12072

19. Dodgson JE, Chee Y, Yap TS. Workplace breastfeeding support for hospital employees. J Adv Nurs 2004;47(1):91-100. https://doi.org/10.1111/j.13652648.2004.03070.x

20. Soomro JA, Shaikh ZN, Saheer TB, Bijarani SA. Employers' perspective of workplace breastfeeding support in Karachi, Pakistan: A cross-sectional study. Int Breastfeed J 2016;11(1):24. https://doi.org/10.1186/s13006-016-0084-7

21. Weber D, Janson A, Nolan M, Wen LM, Rissel C. Female employees' perceptions of organizational support for breastfeeding at work: Findings from an Australian health service workplace. Int Breastfeed J 2011;6(1):19. https:// doi.org/10.1186/1746-4358-6-19

22. US Department of Health \& Human Services. Office on Women's Health. Business case for breastfeeding. https://www.womenshealth.gov/supportingnursing-moms-work/lactation-break-time-and-space-all-industries (accessed 5 February 2019).

23. Witters-Green R. Increasing breastfeeding in working mothers. Fam Syst Health 2003;21(4):415-434. https://doi.org/10.1037/h0089617

24. National Department of Labour, South Africa. Basic Conditions of Employment Act, 1997 (Act No. 75 of 1997). Code of good practice on the protection of employees during pregnancy and after the birth of a child. Government Gazette No. 19453:1441.1997. http://www.labour.gov.za/DOL/downloads/ legislation/Codes\%20of\%20Good\%20Practice/basic-condition/Code\%20 of $\% 20$ Good $\% 20$ Practice $\% 20$ Basic $\% 20$ Conditions $\% 20$ of $\% 20$ Employment $\% 20$ and\%20Pregnancy.doc/view (accessed 5 February 2019).

25. Qwerty Digital with intent. The Digital Landscape in South Africa 2017. A data driven look at South Africa's relationship with digital. https://qwertydigital. co.za/wp-content/uploads/2017/08/Digital-Statistics-in-South-Africa-2017Report.pdf (accessed 8 July 2019).

26. Tsai S. Impact of a breastfeeding-friendly workplace on an employed mother's intention to continue breastfeeding after returning to work. Breastfeed Med 2013;8(2):210-216. https://doi.org/10.1089/bfm.2012.0119 